\title{
ASSISTED REPRODUCTION TECHNOLOGY IN A RESOURCE-LIMITED SETTING
}

\section{A.A.E Orhue (FRCOG, FICS, FWACS, FMCOG, FRSM)}

Professor/ Consultant Obstetrician and Gynaecologist, Department of Obstetric and Gynaecology, University of Benin Teaching Hospital, Benin City, Nigeria

\section{Correspondence:}

Professor A.A.E Orhue

progamme director,

Human reproduction research programme unit

Department of obstetric and Gynaecology

University of Benin Teaching Hospital,

Benin City, Nigeria

Email: aaeorhue@yahoo.com

\section{INTRODUCTION}

The idea of Assisted Reproduction Technology (ART) (which at the moment is the highest break-through in the medical treatment of infertility) in a resource- limited setting, sends a contradictory wave of thoughts as to the desirability and affordability with such limited resources because of the high cost implication. Usually, resource- limited settings refer to low income countries, who are still contending will basic developmental issues like poverty, high unemployment, high proportion of ill health in the populace, over population and poor infrastructure ${ }^{1}$. ART cost several thousands of Euro/dollars in high income countries where it is often a part of the spectrum of management of infertility which is both preventive and curable. In low income countries, infertility is only one of the many important health problems that people face and ART being of such high cost, cannot be a considered method for infertility treatment in view of the serious competing demand on the limited resources. It is often emphasized that the main issue in the resource limited setting is over population crisis rather than infertility whose management for now should be by the much cheaper preventive approaches $^{2-4}$.

The issue of whether or not ART is worth considering at all, for infertility management, in resource limited settings, should not be wished away with the reinstatement that infertility prevention should be the emphasis while the limited resources, should be reserved for solving the over population crisis. Such an approach is rather simplistic and devoid of the knowledge of the premium placed on child bearing in the socio-cultural setting of the people, in these resource limited countries. While the people can easily cope with overpopulation at the individual level, because of the socio cultural care for each other, they cannot cope with infertility. The individual, society and communities are devasted completely by infertility or childlessness.

In Western societies, reproduction is often a self chosen goal and largely a personal choice made by the individual or couple, hence infertility is often a problem at the individual level without societal implications ${ }^{5}$ Infact, there are situations in Western societies, where couple decide not have children in their marriage. In the developing countries, having children is a social obligation, a performance due to the

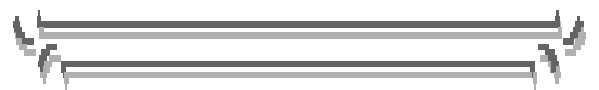


family (in laws) and whole community. Children secure marriage, confer social status, guarantee rights of property and inheritance, assist with labour, offer social security in old age and provide continuity of maintaining family name ${ }^{6-8}$. The main reason for marriage is for child bearing and not love as it is in Western countries. Often, there are no prior love plays or even familiarity between individuals in the marriage which is organised and sponsored by the families, society and community. It is only childbirth that secures the marriage union and bind families and communities together. There can never be a marriage in the developing countries in which the couple decide on voluntary childlessness. Thus, infertility in the developing countries is pervasive and a serious concern especially to the woman leading on to marital disharmony, neglect, abuse, loss of social security, polygamy and even suicide $^{9-11}$.

Infertility in the developing countries is therefore, not just a desire at the level of the individual (as it is in western culture). It is a huge disease burden with devastating consequences on the individual and society that deserve priority resolve amongst other disease chain ${ }^{12}$. This is why infertility management should extend beyond prevention to include comprehensive treatment or else those presently afflicted with the disease will never be helped. Even at this, it is feared that extending infertility treatment to include ART in resource limited settings may be impracticable, for reasons of unstable infrastructure, poor staff morale and poor maintance culture $^{2-4}$. This is why it is assumed paradoxical to contemplate ART is in a resource limited setting with such obvious constrains.

This review shall include a detailed description of ART and the present day indications. The pattern of infertility in the developing countries is critically reviewed against the background knowledge of current socio cultural correlates of infertility in a resource-limited setting to assess the need for ART for infertility management. Finally, the review shall present the outcome of ART in a resource limited setting like Nigeria with emphasis on the practice, prospects and future development of infertility management in developing countries.

\section{Assisted Reproduction Technology (ART) Basic Knowledge}

Assisted reproduction technology encompasses several techniques to by-pass the problems preventing conception through the normal sexual intercourse in a stable relationship. These techniques involve either artificial insemination or artificial fertilisation in which there is alteration in the inherent process leading unto conception and without sexual intercourse. There are several types of ART presently viz:

\section{In vitro fertilization and Embryo Transfer (IVF and ET)}

This refers to the assemblage of procedure culminating in the retrieval of human Oocyte which is approximated to potentiated Sperms in a test tube (In vitro) in a laboratory condition equilibrated to the same physiological condition as obtains in the normal human environment (in vivo). It thus involves artificial fertilisation outside the human body and without sexual intercourse.

\section{Gamete intra-fallopian tube Transfer (GIFT)}

This involves the transfer of Oocyte and potentiated sperm to the fallopian tube with the laparoscope for in vivo fertilisation and subsequent 
implantation and pregnancy. Although this does not also involve sexual intercourse, the artificial fertilisation occurs in vivo within the human body.

\section{Zygote intra fallopian tube transfer (ZIFT)}

This involves the transfer of zygote (from fertilisation of Oocytes obtained through IVF and potentiated sperm) to the fallopian tube with the laparascope. The fertilisation is done in vitro before the transfer and without sexual intercourse.

\section{Intra uterine Insemination (I.U.I)}

This involves the transfer of potentiated sperm using special insemination catheters through the cervical os into the uterine cavity using aseptic technique at the peri- ovulatory period which commonly nowadays is preceded by a super ovulation treatment (with gonadotrophin and human chorionic gonadotrophins to trigger ovulation) to facilitate release of several Oocytes to enhance the prospect of conception. It does not involve sexual intercourse but the artificial fertilisation occurs within the human body (in vivo). It should be noted that insemination of sperm at the cervix or unto the cervical os only, with whole or partially treated sperm is not grouped as Assisted Reproduction because it does not alter the inherent process leading unto conception.

\section{Other complimentary procedure to I.V.F.}

\section{(1)}

spermatozoon obtained from the epididymis may be used. It involves directly injecting a single spermatozoon, unto an Oocyte using special equipment (The ICSI machine). This is a major break-through in the treatment of male infertility.

\section{(2) Cryo preservation and} Transfer of Embryo

When there are more embryos than can be transferred in any cycle, the excessive embryo are cryopreseved by freezing in liquid nitrogen. When the frozen embryos are required later, they are thawed, and transferred into the uterus. This helps to prevent repeating the stimulation for Oocytes again. At times cryopreservation of embryo is planned when there are contraindications to embryo transfer as in fear of severe Ovarian Hyperstimulation Syndrome (OHSS) or there has been an inadequate endometrial response.

\section{In Vitro Fertilization and Embryo transfer - Basic facts and indications}

The first successful IVF in human, was in July 1978, leading to the birth of Louise Brown in Great Manchester City U.K $\mathrm{K}^{13}$. The IVF cycle was performed in a natural cycle in which the naturally ovulated Oocyte was retrieved with the laparoscope under G.A. The IVF was performed because Lesley Brown had no tubes (which had been removed from two previous ectopic) but had normal uterine cavity and was normally ovulating. Hence the IVF by passed the damaged tubes to obtain the 
ovulated Oocyte and approximate it to the sperm in vitro to obtain the embryo which was also transferred under general anaesthesia into the uterine cavity and subsequently pregnancy occurred. Without IVF Lesley Brown's chance of childbirth was zero.

In the early days, IVF was performed in natural cycles for women with damaged tubes but with normal uterus and ovulatory cycles. Later, it was found also helpful in other situations with normal tubes but with anovulation. A major break through occurred in the area of male factor treatment with the introduction of the Intracytoplasmic sperm injection $(\mathrm{ICSI})^{14}$. With the technique of pre implantation genetic diagnosis and embryo selection, healthy babies can now be possible by avoiding transfer of embryo with genetic disease ${ }^{15}$, and with embryonic stem cell research new areas have been opened up ${ }^{16}$.

The indication for assisted reproduction has now greatly expanded to include Oocyte donation to treat premature menopause ${ }^{17}$ surrogacy for women with damaged uterus $^{18}$, effective endometriosis treatment $^{19}$ and even assessment and diagnosis of male factor ${ }^{20}$.

\section{IVF Procedure}

At the onset, IVF was performed in the natural cycle of the woman and several days were often spent performing serial laboratory test to ascertain when ovulation will occur for laparoscopic retrieval. Often, one Oocyte was retrieved for fertilisation and therefore one embryo transferred. The success rate for this natural cycle I.V.F. was less than $5 \%$ and required tedious laboratory test and clinical skill in laparoscopic surgery. As ovulation stimulation drugs were discovered, IVF cycles were then stimulated, first with Clomiphene citrate but later with gonadotrophin in order to generate many Oocytes and this was associated with much greater pregnancy rate re $^{21-23}$. The main problem at this period, with stimulated cycles, either with clomiphene or gonadotrophins, was premature ovulation leading often to failed Oocyte retrieval but that was resolved with the introduction of L.H.RH analogues for pituitary down regulation before stimulation with HMG 24,25 . Currently IVF procedure consist of series of steps as explained below.

\section{Controlled ovulation stimulation}

This involves the prior use of LHRH analogue for pituitary down regulation followed by gonadotrophin injection (HMG) aiming for multiple follicular growth and development and HCG administration at the appropriate time as the trigger for ovulation. This is orderwise referred to as super ovulation and is a process whereby ovulation is deliberately programmed in a woman who may have been ovulating albeit, irregularly or a woman who is Anovulatory. The aim is to stimulate the development of several mature follicles from the ovary in order to increase the chances of obtaining fertilisable eggs $^{26}$. The use of the LHRH analogue prevents premature ovulation (which may cause failed oocyte retrieval) and the $\mathrm{LHRH}$ analogue and gonadotrophins are administered till day of the HCG injection. The follicular growth is currently monitored with trans-vaginal ultrasound combined in appropriate cases with serum oestrogen measurement. ${ }^{27}$.

\section{Oocyte retrieval}

Once the follicles have reached the appropriate size of about $18 \mathrm{~mm}$, Oocyte retrieval is performed now with transvaginal ultrasound using ultrasound guided probe in which only local analgesia like para cervical block is used on out patient basis ${ }^{28}$. This has 
made the procedure almost risk free and without stress to the patient.

\section{Examination; fertilization and incubation}

Once the follicles are retrieved, the fluid is examined and the oocytes are removed. The damaged or malformed ones are discarded and the others are washed and placed in Petri dishes with nutrient solution. After-wards, the Oocytes are mixed with the potentiated sperm from the male partner and equilibrated under laboratory condition to allow fertilisation to occur usually within 24 hours. The fertilised Oocyte (embryos) are then moved into an incubator for a few days to allow the cells to divide further.

Meanwhile, the woman is administered with progesterone injection often from the day of Oocyte retrieval to prepare the uterus for implantation of the embryo. In the older women (particularly recipients of oocyte donation) the progesterone administration may have began from the day of HCG administration to the oocyte donor to facilitate better endometrial preparation in the recipient. Nowadays, instead of progesterone injection, macronised progesterone pessaries (Gestones), as vaginal inserts, are used to minimise the risk in injection treatment. However, most women cannot tolerate the vaginal pessaries because of sourness in the vagina from trauma of transvaginal needle puncture in the first 5-7 days following the Oocytes retrieval. This is why there is still recourse to the progesterone injection commonly in the first one week. Thereafter, the progesterone pessary is often used for luteal support for the next $10-12$ weeks for those who achieve clinical pregnancy.

\section{Embryo Transfer}

At the 2-8 cell stage the embryo with the best chance of implanting are transferred into the uterine cavity through the undilated cervical os on the $3^{\text {rd }}$ day following oocyte retrieval using a special embryo transfer catheter.

This is performed using aseptic technique under a sterile operation theatre condition. The procedure should be free of any difficulty in the identification of the cervical os, passage of the transfer catheter through the os and release of the loaded embryo into the uterine cavity which is accomplished without the catheter touching the uterine fundus or walls. Difficulty with transfer reduces the chances of successful implantation and pregnancy. Although embryo transfer is commonly performed clinically, the process can be assisted with ultrasound to guide the placement of the catheter before the release of the embryo. As much as three (but less commonly now days) four, embryos are transferred to increase the chances of implantation especially in the older women. Presently, the consensus is for the transfer of one or two embryos especially in the young woman as a way to reduce the common complication of multiple pregnancies in $\mathrm{IVF}^{29}$. The remaining embryos not transferred are Cryo preserved for later use. Once an embryo implants, development continues normally in the absence of spontaneous abortion or any other complication.

\section{Core issues in Human Reproduction and role of IVF.}

The core of the reproductive process is the union of the sperm and oocytes. This union occurs often and normally naturally, at the outer third of the fallopian tube. Thus, an essential feature of this reproductive process is the adequate and appropriate 
transport of the oocyte (after release from the ovary) to the outer third of the fallopian tube to be fertilised by the sperm which had found its way to the outer fluid of the tube after it had been deposited into the vagina. Hence, release and transport of the oocyte and sperm from the point of release are essential steps for the union of the oocyte and sperm for fertilization. Because, the sperm has a longer distance to transverse (from the vaginal to the outer third of the fallopian tube) motility is thus the most important quality required of the sperm to succeed in the task of union and fertilisation.

In practical realities, coital frequency is the deciding factor for the natural rate of pregnancy which means the higher coital frequency is associated with more pregnancy rate though a coital frequency of four or more per week may be associated with exhaustion in the older couple ${ }^{30}$. The idea coital Type of single treated infertility Causal factor

1. Anovulation (Amenorrhea; Oligomenorrhea; or normal periods).

2. Unexplained infertility

3. Sperm Disorder with Normal count

4. Sperm Disorder with low count

5. Tubal Damage frequency is 2-3 times per week evenly spaced at every 48 hours apart ${ }^{30}$. In normal couples, pregnancy rate per monthly cycle with idea coital frequency is $20-25 \%$ but in six month pregnancy rate will increase to $50 \%$, in one year it is $90 \%$ and in two years it is $96 \%^{31}$. In this context, some couples fail to conceive by chance and even success, naturally or from treatment can never be guaranteed ${ }^{31}$. The truth is that the human reproduction process is very inefficient with as low a pregnancy rate per monthly cycle of $25 \%$ in the ovulatory cycle of a young woman less than 30 year. For the older woman, even with ovulatory cycle pregnancy rate falls to about $12 \%$ per month.

This level of inefficiency is also greatly extended to poor conception rate in couples who had successful treatment for single cause of infertility with the conventional method, as shown in the table.

\section{Conception rate as percentage of couple}
1 Cycle
6 Cycles
12 Cycles

$12 \%$

$65 \%$

$80 \%$

$10 \%$

$40 \%$

$55 \%$

$5 \%$

$10 \%$

$20 \%$

$1 \%$

3\%

$6 \%$

$6 \%$

$10 \%$

$18 \%$

(Cummulative conception rate from conventional management in couple with a single cause of infertility. Adopted from Hull MGR et al '85 (32).

As shown from the table above, in all of the causal factors, the best conception rate of $12 \%$ and $10 \%$ was from Anovulatory and unexplained infertility in one cycle while all other factors in 1 cycle have a near chance pregnancy rate of $6 \%$ or less ${ }^{32}$ 
It is quite obvious that left with the conventional infertility management methods, the inefficiency of the human reproduction capacity cannot be improved much even with the best of treatment. For some of the causal factors like sperm disorders and tubal damage, conventional infertility treatment does not offer better than chance occurrence of pregnancy per cycle; hence the need for Assisted Reproduction Technologies like IVF and ET. The technique of IVF allows a by-pass of some (if not all) of the insurmountable infertility causal factors by the conventional methods to allow pregnancy and child birth where order wise the chances of birth is zero. The problems preventing the union of oocytes and sperm in the natural setting is by-passed by the super ovulation, oocyte retrieval, fertilisation and incubation and subsequently the Embryo transfer. In good IVF centres, the pregnancy rate equates to the natural pregnancy rate per monthly ovulatory cycle of $25 \%$. At times the IVF success rate is much higher than $25 \%$ because of multiple embryo transfer which allow the conception rate to surpass the natural rate which often is monofollicular and uni-embryo implantation $^{33 ;}$ 34. There is no other method of infertility treatment currently, that can achieve this same pregnancy rate per month of $25 \%$ apart from IVF. Like other methods of treatment, repeated IVF has a cumulative success rate which is virtually the same, $25 \%$ or more, for every attempt for at least the first 6-8 repeat cycles ${ }^{35}$ This means a higher success rate with repeat IVF attempt.

\section{Problems and Risks of IVF}

\section{Problems}

1. Technique is complex, expensive and stressful. The cost is $\$ 10,200$ in USA per cycle and in Britain, it is four to five thousand pound sterling per cycle. The high cost is due to the high tech nature of the equipments, cost of the drugs, and media, and manpower cost.

2. The $25 \%$ pregnancy rate per cycle implies there is $75 \%$ failure rate. However, it is often not recalled that the natural pregnancy rate per month for a young ovulatory woman under 34 years is similarly $25 \%$ with $75 \%$ as failure rate.

\section{Complications of IVF and ET}

1. Ovarian infections, Ovarian rupture though rare, may occur

2. Ovarian Hyperstimulation syndrome (OHSS). This may occur in $(0.6-14) \%$ of IVF treated population ${ }^{36}$. It is often a serious life threatening complication. The condition commonly occurs with the use of $\mathrm{LHRH}$ analogues and HMG followed by H.C.G. as trigger for ovulation. Polycystic ovary and administration of HCG appear to be a major predisposing factor for development of the full blown OHSS. Hence preventing OHSS at times involves non use or reduction in the dose of HCG or astute careful retrieval of all the oocytes followed by fertilisation and then cryopreservation of the embryo for transfer later in a non-stimulated cycle when the OHSS has subsided $^{37}$. In clinical practice, the predisposing factors may have been missed or were absent in the particular individual. The full blown OHSS disease many manifest (in the severe form) anytime from the $2^{\text {nd }}$ or $3^{\text {rd }}$ or

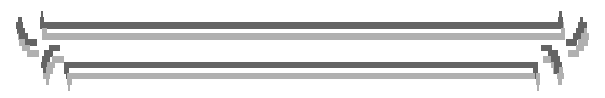


even $5^{\text {th }}$ day after embryo transfer with abdominal pain, abdominal distension, vomiting and respiratory difficulty associated with high packed volume level. Often, astute and aggressive treatment is required to forestall the disease process hence it is always better prevented by all means.

3. Risk of G. A., where it is used for any of the IVF procedure.

4. Spontaneous abortion: This tends to be higher following IVF pregnancies due to factors like poor embryo malimplantation and aging of the woman.

5. Ectopic pregnancy and at times heterotrophic pregnancy. This is often a serious problem that requires urgent attention

6. Multiple pregnancy: This complication occurs in almost $30 \%$ of IVF pregnancies. Often due to transfer of several embryos as a way to ensure implantation and pregnancy. Approach nowadays is to transfer only one embryo for the young woman and not more than two in the older woman.

7. Preterm delivery: This may be due to the multiple pregnancy or gross anomaly in the fetus. Adequate preparation to handle this problem with the provision of an effective and efficient neonatal intensive care unit at I.V.F. centres is important as a solution to this problem.

\section{Pattern and trend of infertility world wide}

The level and pattern of infertility vary greatly depending on the prevalence of "Core infertility" (biological variation in chromosomal, congenital and endocrinological abnormalities) and "acquired infertility" (geographical variation in infectious, environmental and occupational factors) $)^{38 ; 39}$. Apart from this; the reports of causes and outcome of infertility treatments is highly variable because of the lack of a standard protocol for the investigation and treatment which makes it difficult to compare results with respect to patterrn; differences and similarities. The WHO produced a standard protocol for the investigations of the infertile couple ${ }^{40}$ which was tested in 8,456 couples from 34 centres in 25 countries world wide including four centres in Africa. The protocol standardised procedure, definitions and identification of the factor that are important in assessing the fertility status in both the males and females. The results of the study which was published in 1985 showed the pattern and trend of infertility world wide in the developed and developing countries ${ }^{41}$. African countries amongst all other developing countries were found to be peculiar in several respects, viz:

i. African women had infertility for longer duration before consulting for assistance.

ii. African women were much younger than their male partners almost like the men were marrying women, of the age of their grandchildren.

iii. African women had much higher past history of S.T.D. and prior pregnancy complication.

iv. African women had much higher secondary infertility than primary infertility.

The conclusion from the study was that infertility in African countries, were essentially due to sexually transmitted diseases and complications following induced abortions and poor maternity care which are all preventable by simple strategies ${ }^{42}$.

It was then recommended that approach to infertility management in

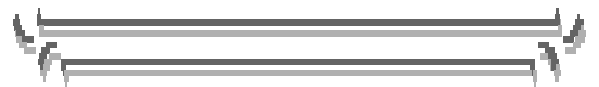


the African countries should be preventive. The issue of Assisted Reproduction Technology (ART) as treatment for infertility was emphasised as being of the least priority importance in infertility management.

\section{Current Status of infertility in} developing countries

The W.H.O. study published in 1985 involved four African countries in which 842 couples with different social and cultural background were evaluated. The studies were performed in a polyclinic setting in which the same areas at the participating centres were used for various other purposes other than for the management of the infertility alone. In contrast, several of the other participating centres in Europe but to a lesser extent in Asia and Latin America, managed the infertility couple in their studies from centres that were dedicated for infertility because of the high premium on infertility as a reproductive health index. Such dedicated infertility programmes are uncommon in most African countries and certainly did not exist in all the African centres who participated in the W.H.O. studies, even though there is a huge burden of infertility in the gynaecological practice.

This would suggest that the findings in the socio-cultural and epidemiological correlates of the infertility in the W.H.O. studies may not have been truly comparable; unless African studies were performed in similar dedicated infertility programme. This is perhaps why African countries had strikingly different infertility findings as highlighted in the W.H.O. study. Perhaps, the findings from the WHO study if performed in similar dedicated fertility centre as in Europe etc, may have found other infertility causes that are not preventable by the recommended strategy. The $\mathrm{WHO}$ recommendation of prevention as the most cost effective approach to infertility management in African countries would mean, that the prospect of infertility management in contemporary times of concurrent preventive and curative approach including with ART will never gain government support, for public funding. This is the reason for low public spending on infertility management in the developing countries. The findings from studies of the socio-cultural and epidemiological factors of infertility from a dedicated program of infertility in Nigeria did not corroborate several aspects of the prior WHO study of $1985^{41}$. Thus, the prospect of infertility management in a developing country attaining the status as in developing countries is best illustrated by the experience at a unique programme for infertility management in a University hospital in Nigeria.

\section{A University hospital based infertility programme in Nigeria}

The infertility programme is part of the department of obstetrics and gynaecology of the University of Benin Teaching Hospital Benin City, Edo State, Nigeria and is called the Human Reproduction Research Programme (H.R.R.P.). The programme has its own site building with consulting rooms, laboratories and administrative office space. Consultation for infertility is daily during the working period and the programme has its own Nurses, Laboratories Scientist, Administrative and Support staff. Since the Programme has its own building with ample space, clients are received at the centre as couples and given assurance of strict confidentiality of information even between consorts. After the initial consultation, follow-up visit are tailored for the convenience of the couples without the usual

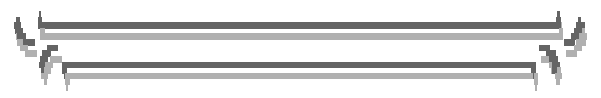


institutional rigidity but within the working period. Good rapport was commonly established between the couple and clinic staff after one or two visit with same staff (Nursing and Medical) unlike consultation in the routine gynaecology clinic where clients encounter different staff at each visit. This management approach encouraged the women to volunteer confidential information and truth about their age, parity and husband occupation.

The main tool for the investigation, classification and diagnosis of infertility was the World Health Organisation (WHO) format for the investigation of the infertile couple as published in 1984 by Rowe and Farley ${ }^{40}$. All the couples were assessed by the consultants and by specially trained Nurses. After full investigation using the WHO protocol, diagnosis was established and the conventional treatment of infertility provided. The couples were followed up thereafter for pregnancy outcome. The results from the programme assessed whether the local pattern of infertility are a reflection of the WHO study over 2 decades ago on which findings and recommendation on infertility management, strategy for African countries was based.

\section{Deduction from the University hospital based infertility Programme in Nigeria}

The outcome of 10 years of infertility management involving almost 2000 consecutive couples at the programme was recently published ${ }^{43}$. Although findings suggest infertility pattern similar to the prior WHO study, there are several differences. The Nigeria woman was not so much younger than her husband, the secondary infertility rate was $85.7 \%$ with primary infertility rate of only $14-3 \%$ and complications of induced abortions contributed the highest to tubal blockage, (the commonest female causal factor) followed by vaginal deliveries attended by unskilled persons in unhygienic environment. These are all very different from the prior WHO study. It also showed that there are infertility causes that are unpreventable.

The differences in this study and others is due to the assurance of confidentiality and rapport established between patients and programme staff which led to the disclosure of the truth about the women age, parity and details of the mode and place of delivery. Culturally Nigerian women reduce their age; deny prior abortion and deliveries especially in previous relationships (because they must be seen as young and innocent of prior sexual relationship) and do not disclose the mode and place of delivery. The rapport and confidentially essential for disclosure may not have been possible in the environment described in the $\mathrm{WHO}$ and other studies which may explain the marked discrepancy between this study and other studies of infertility.

The pregnancy rate of $40.1 \%$ occurred mainly from the treatment of female factors alone and spontaneous pregnancy in $7.5 \%$ attributable to the education and counselling on coital timing provided at the centre. Pregnancy rate was very poor in the female factors requiring surgery, in several of the moderate to severe male factor alone but particularly in couples with both male and female factors.

Thus, in almost $60 \%$ of the study population, the infertility program (representing the highest concentration of resources in a developing economy for most types of infertility treatment) could not help the couple achieve pregnancy and further treatment improvement is impossible with the conventional method 
available. This is principally because a sizeable proportion of the infertile couples had both female and male factors and a sizable proportion with female factors required surgical treatment that were associated with poor outcome. These, are intractable to conventional infertility treatment method and are best treated with Assisted Reproduction Technology (ART).

The capacity to treat aspect of infertility unresponsive to conventional methods would improve results and therefore justify the investment. Hence the data from this study is a case for implementing ART as a key element in future infertility research and treatment. This study outcome is proof that conventional infertility treatment method has insufficient capacity for treatment of the majority of infertile couple in our environment.

$\begin{array}{llr}\begin{array}{l}\text { Preventive } \\ \text { approaches versus } \\ \text { management }\end{array} & \begin{array}{r}\text { Curative } \\ \text { infertility }\end{array} \\ \text { economy } & \text { a } & \begin{array}{r}\text { developing } \\ \text { dhe issues }\end{array} \text { raised in the }\end{array}$
recommendations of ART as back up infertility treatment deserve further discussion. The data from our study confirm that secondary infertility is the main issue and can be reduced by preventive strategies like access to effective family planning, improved maternity care and Health education to reduce risky behaviour that can cause S.T.D. Not only is prevention cheap but the strategy will confer several other benefits to women's right and society, hence radical proponents of preventive strategy for infertility management, recommend total commitment of resource only to prevention $^{44-46}$. This radical stance is untenable in contemporary times, because in the case of disease control with HIVIAIDS there is free distribution of antiretroviral drugs to HIV Positive patients (instead of allowing those afflicted with HIV Virus to progress to AIDS and die) in addition to huge money spent on the campaign to promote the use of condom as prevention for the disease ${ }^{47}$.

No matter the degree of coverage of any preventive strategy it will not apply to the several people who already now are infertile and also it is not all infertility cause that are preventable ${ }^{48}$. A much better approach is the moderate view of infertility prevention advocating that priority should be accorded prevention, but adequate resources should be directed to curative measures to benefit the population already afflicted with infertility $^{49-51}$.

The other issue against curative measures for infertility is the over population issue because there is the demographic paradox that countries with the highest overall fertility rate are also those in which the prevalence of secondary infertility is highest ${ }^{52}$. There is therefore strong argument against infertility treatment especially with extension to ART because it would be wrong use of scare resources and worsening of the world population crisis. This over population as a reason to neglect infertility is wrong logic seeking for the few infertile women to bears the burden of world over- population in which their individual liberty for child wish is unjustly denied ${ }^{51,53,54}$. More over, such an approach is highly oppressive of those who are infertile as there are better ways of population control which do not infringe upon peoples' right like educating women, providing contraception and safe abortion service $^{47}$. More recently, it was suggested that the over population problem of the world is not any more due to high fertility rate (whether in the developed or developing countries) but due to increased life expectancy 
causing population growth by population aging ${ }^{54}$.

It is therefore reasonable to suggest that for the developing economy the infertility management approach should be concurrent preventive and curative. The data from our study at the infertility programme has now defined the content of effective curative infertility management to include establishing infertility programme with competence for ART treatment because majority of the infertility causal factors are intractable to conventional treatment. Certainly the social, emotional, physical and economic consequences that infertile couple (and in particular women) face, justifies investing in treatment options like ART in developing countries ${ }^{56 ; 57}$ This was our impetus for the up grading of the infertility programme to include capacity for ART services.

IVF in Public Hospital in Nigeria: Practice, problems and prospects After almost two decades of infertility management at the infertility programme, it was found that almost $60 \%$ of the couple could not achieve pregnancy. This is because of causal factors which are intractable to conventional methods occurring singly or in combination. In order to improve the treatment outcome, the programme was upgraded to include facilities for Assisted Reproduction technology. The programme site building was expanded to provide more space for office hysteroscopy, ultrasound with transvaginal probe and full in vitro fertilization and embryo transfer (IVF and ET) equipments and intracytoplasmic sperm injection (ICSI) machine. Full ART services including intra uterine insemination (I.U.I) and IVF and ET with ICSI began June 2007.

IVF and ET with ICSI was offered to the couple who could not achieve pregnancy by the conventional method. The IVF and ET approach involved the standard practise of pituitary down regulation, followed by super ovulation with ganodotrophin using the long protocol during which follicular response was assessed by regular transvaginal ultrasound. Human Chronic Gonadotrophin (HCG) was administered for ovulation induction when follicles of at least $18 \mathrm{~mm}$ diameter were obtained and Oocyte retrieval by the transvaginal route performed 35-36 hours after the HCG. The Oocytes were assessed by the embryologist, fertilised with the previously potentiated sperm and resulting embryo transferred on the third day trans- cervically using standard embryo transfer catheters. In the appropriate cases ICSI was performed when there was significant male factor. The IVF method followed the standard Western method as variously published ${ }^{58-61}$.

The IVF service delivery was done in batches of $30-40$ couples as a deliberate policy and involved the prior synchronisation of the menstrual cycle of the women at times across one to three cycles with sequential oestogens and progestogens. This allowed the focalisation of all the manpower and infrastructure for the given period of Oocyte retrieval, embryo culture and transfer. It also allowed the concentration of work for a stipulated period for appropriate manpower deployment. This way, the muted fears of unstable light and water and poor staff motivation in the public hospital system was resolved because during any IVF run adequate staff and stable infrastructure is assured.

The outcome of the first year experience with IVF in a public hospital has been collated and is being published elsewhere ${ }^{62}$. There were 150 couples handled in 5 batches in which there were about 30 couples per 
batch. The mean age of the women was 36 years witha range of $32-44$ years. The mean parity was 0.7 and mean duration of infertility was 10.5 years. The women had several previous failed treatment mainly surgery (see table 2) and the commonest indication for the IVF was tubal disease and combined male and female factors. The clinical pregnancy rate was $28 \%$ per cycle in which there have been 8 deliveries so far. This result is comparable to other I.V.F centres $^{63-65}$. The most severe problem encountered was severe Ovarian Hyperstimulation syndrome (OHSS) which occurred in two women in the series and resolved with standard management as described by Lainas $2002^{65}$.

Thus IVF Service delivery in a public hospital system in Nigeria is practicable and effective as a back up for infertility cases that are intractable to conventional treatment as in almost $60 \%$ of the infertile population. For the women who achieve pregnancy by this method there were are no other ways to assist them and hence ART is a worth while investment even in our resource limited environment. The IVF and ET programme adopted the full Western style approach and achieved a pregnancy rate that is comparable to other IVF centres world wide. This fact has now broken the myth that IVF is not possible in a public hospital system as suggested by other workers ${ }^{67,68}$. The strategy of batching allows the optimisation of the manpower and focused infrastructural support during an IVF run. Aside from this, batching also allowed the sufficiently large number of couples such as 40 as in our case in a single run which is useful for research and teaching the IVF knowledge. Having now established the structure for IVF at the programme, the next stage is to assess how the cost of the IVF can be reduced to make it more affordable. This will involves reduction of cost of drugs, equipments and substantial reduction in the chargeable fee by the hospital as advocated by other workers ${ }^{50,63,64}$. It is when the cost of IVF is affordable that a truly comprehensive curative infertility treatment can be said to be available and comparable to standard of care for infertility as it is in the developed economy.

\section{Conclusion}

Infertility in developing countries is pervasive and has devastating consequences for the couple especially the woman who culturally is held culpable for the cause of the infertility. Since childbearing is the sole purpose of marriage in these settings, infertility or childlessness leads to societal disharmony, divorce, violence, suicide and other psychological disorders. It is in every way a disease burden that deserves priority attention because of the widespread effect on society and the community. This is in contrast to infertility in the Western society where the effect on society is minimal if at all, and yet infertility treatment at whatever cost including ART is never denied anyone who required it.

In the developing economy, infertility management has been advised to adopt the much cheaper preventive approaches because of limited funds and burden of over population. Assisted reproduction technology (ART) for infertility management, is said to be wasteful and not practicable because of poor public infrastructure low man power capacity and low staff morale. Recent studies have shown that in $60 \%$ of infertile population in some developing economy, the effective treatment is ART or most perhaps will never achieve conception. The report of IVF in a public hospital with a comparable pregnancy rate like

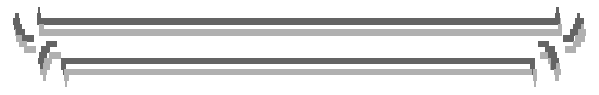


others IVF centres world wide confirms that IVF in a public hospital setting is practicable and worth the investment in a developing economy. What remains is the approach for IVF cost reduction to make it affordable. This is the only way that contemporary concept of infertility management will have prospect in a developing economy to be practiced appropriately in the context of the preventive and curative, including ART.

\section{REFERENCES}

1. Van Balen F. In voluntary childlessness: a neglected problem in poor resources areas. Hum. Reprod. ESHRE monography 2008.

2. Sheth SS, Malpan AN. In appropriate use of New Technology: impact on women's Health. Int. J. Gynaecol. Obstet. 1997, 58: 159-165.

3. Okonofua FE. the case against New Reproductive Technologies in developing countries. Br. J. Obstet Gynaecol. 1996: 103: 957967.

4. Okonofua FE. New Reproductive technologies and infertility treatment in Africa (Editorial) Afr. $\mathrm{J}$. Reprod. Health 2003; 7.

5. Pennings G. Ethical issues of infertility treatment in developing countries. Hum. Reprod. ESHRE monograph 2008; 15-20.

6. Dyer SJ. the value of children in African countries insights from studies on infertility. J. psychrosom. Obstet Gynaecol. 2007; 28: 69-77.

7. Sunby J. Infertility in the Gambia: Traditional and Modern Health care. Patient Edc. Comms 1997, 31; 2937.

8. Richards SC. Spoiling the womb: definition aetiology and responses to infertility in North west province Cameroon. Afr. J. Repro. Health, 2002: 6; 84-94.

9. Van Balen F. Gerrits T. Quality of infertility care in a poor resource area and the introduction of New Reproductive technologies. Hum. Reprod. 2001: 16: 215-219.

10. Ebomoyi E, Adetoro OO. Socio-biologic factors influencing infertility in a rual Nigeria Community. Int. J. Gynaecol. Obste. 1990; 33; 41-47.

11. Hollos M. Profile of infertility in southern Nigeria: a women voices from Makin. Afr. J. Reprod Health 2003; 7: 46-56.

12. Akande EO. Affordable Assisted Reproductive Technologies in developing countries: PROS AND CONS Hum Reprod. ESHE Monograph 2008: 12-14.

13. Steptoe PC, Edward RG. Birth after re-implantation of a human embryo. Lancet 1978. 366. 
14. Palermo $\mathrm{G}$ et al. Pregnancies after Intracytoplasmic injection of single spermatozoon into an oocyte. Lancet 1992: 340, 17-18.

15. Handyside AH. et al: Pregnancies from biopsies human pre implantation embryo sexed by Y-specific DNA amplification. Nature, 1990: 344: 768-770.

16. Reubinoff BE. et al: Embryonic stem cell lines from human blasto cysts: somatic differentiation in vitro. Nature Biotechnology 2000, 18: 399, 404.

17. Serhal PF, Graft IL. Ovum donation - a simplified approach. Fert. Steril 1987; 48; 265-269.

18. Utiam WH, Sheehan L, Gold Farb JM et al. Successful pregnancy after in vitro fertilisation and embryo transfer from an infertile woman to surrogate. $\mathrm{N}$. Engl. J. Med. 1985; 313; 1351-1352.

19. Oehninger, S; Acosta, A.A; Kreiner, D. et al: In Vitro Fertilisation and Embryo transfer (IVF/ET): An established and successful therapy for endometriosis. J. In Vitro. Fertil. Embryo trans 1988; 5: 249.

20. Acosta AA, Oehninger $S$, Morshedi Swanson RJ, Scot $R$, Irianni F. Assisted reproduction on the diagnosis and treatment of male factor: Obstet. Gynaecol. Surv. 1988; 44:1.

21. Trownson AO, Leeton JF, Wood C, Webb J, Wood J. Pregnancies in human by Fertilisation in utero and embryo transfer in controlled ovulatory cycle. Science 1981: 212: 681.

22. Edwards RG, Steptoe PC. Current status of in vitro Fertilisation and implantation of human embryo. Lancet 1983; 2: 1265 - 1270.

23. Jones HCV Jr, Acosta AA, Andrew MC. Et al: three years of in vitro Fertilisation at Norfolk. Fertil. Steril 1984; 42; 826.

24. 'Fleming $\mathrm{R}$, Adams $\mathrm{A}$, Barlow DA, Black W, McNorton MC, Courts JRT. A new systematic treatment for infertile women withabnormal hormone profile. Br. J. Obste Gynaecol. 1982; 80-83.

25. Porter R, Smith W, Graff IL, Abdulwahid $\mathrm{N}$, Jacobs $\mathrm{H}$. Induction of ovulation for in Vitro fertilisation using Buserelin and gonadotrophins Lancet 1984; 2; $1284-1285$.

26. Cooke ID. Infertility. In Kath Edmonds (eds) Dewhurst's Textbook of Obstetrics and Gynaecology for Postgradsuates $6^{\text {th }}$ Edition. Oxford London, Blackwell Scientific 1999; 432-440.

27. Hackeloer BJ, Fleming, Robinson HP, Adams AJ,

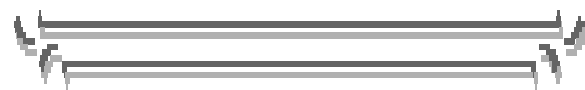


Coutts JR. Correlation of ultrasonic and endocrinologic assessment of human follicular development. Am. J. Obstet. Gynaecol. 1979; 135; 122-128.

28. Wikland $M$, Nelson $L$, Hansson R, Hamberger L, Janson PO. Collection of Human oocytes by the use of sonography. Fertil. Steril, 1983: 39: 603.

29. Flisser E, Licciardi F. One at a time: Fertil Steril 2006; 85 555-558.

30. Kanthi Bansal. In: Kanthi Bansal (eds) Practical approach to infertility management. Jaycee medical publishers. New Delhi 2004: 3-5.

31. Hull MGR. Indication for assisted conception. In. Edwards R.C. (eds) Assisted human conception. British medical Bulletin series. Churchill Livingstone 1990; $580-595$.

32. Hull MGR, Gilazener CMA, Kelly NJ. et al: Population study of causes treatment, and outcome of infertility. Br. M. J. 1985: 291; 1693

33. Fathala MF. Current challenges in Assisted Reproduction In: Vayena E; Rowe PJ, Griffin PD. (eds) Current practice and controversies in Assisted reproduction. W.H.O.

Geneva 2002: 2-12
34. Society for Assisted Reproductive Technology and the American society for Reproductive medicine: Assisted reproductive technology in the United State and Canada: 1995 results generated from the American society for reproductive medicine/society for Assisted reproductive technology registry. Fertil Steril 1998: 69; 389-398.

35. F.I.V.N.T (de Monson J. et al). Analyse de resultants: 1986 Generalities. Indications, stimulations, range delatentative, age dela femine. Contraception. Fertilitew, sexualite 1987; 15: 740.

36. Rizk. B, Smitz. J. Ovarian Hyperstimulation syndrome after super ovulation for IVF and related procedure. Hum Reprod 1992: 7: 320-327.

37. Fisk NM. Multiple pregnancy. In Edmonds. K. D. (eds) Genohust's Textbook of Obstetrics and Gynaecology for Postgraduates. $6^{\text {th }}$ Edition, Oxford, London, Blackwell Scientific Ltd. 1999; 298307.

38. Belsey, MA. The epidemiology of infertility: a review with particular reference to sub-Sahara Africa. Bull, WHO 1976: 54: 319-41.

39. Sherris JD, Fox G. Infertility and sexually transmitterd disease: A public Health 
challenge population Rep 1983 (L-4) 114-115

40. Rowe PJ, Farley TM. The standard investigation of the infertile couple. In Rowe PJ; Vikhlyaeva EM. (eds) Diagnosis and treatment of infertility. Toronto, Ontario Canada. Haris Huber Publishers 1984: 15-39.

41. Cates W, Farley TM, Rowe PJ. Worldwide parttern of infertility: Is Africa different? Lancet 1985. 1: 596-598.

41. World Health Organisation: Infections, pregnancies and infertility: Perspective on prevention. Fertil Steril: 47: 964-968

42. Orhue A, Aziken M. Experience with a comprehensive University hospital based infertility programme in Nigeri. Int. J. Gynaecol. Obstet. 2008: 101: 11-15.

44 Okonofua FE. The case against new reproductive techologies in developing countries. Br. J. Obstet 1996: 957-967.

45. Sheth SS, Malpani AN. In appropriate use of New Technology: impact on women's Health. Int. J. Gynaecol. 1979: 58: 159165.

46. Okonofua FE. New Reproductive technologies and infertility treatment in Africa (Editoria) Afr. J. Reprod. Health 2003: 7.
47. Pennings G. Ethical issues of infertility treatment in developing countries. Hum Reprod. ESHE monograph July 2008: 1519.

48. Verweij $M$. preventing disease. In: Ashcroft R.E.; Dawson $\mathrm{A}$; Draper $\mathrm{H}$; McMillian JR. (eds) Principles of Health care ethnics, $\quad 2^{\text {nd }} \quad$ Chrichester John Wiley and son 2007, 557-562.

49. Toubia NP. Social pressure is not the only reason people want children. Reprod. Health Matters 1994: 4: 9495.

50. Luna F. Assisted Reproductive Technology in Latin America: Some ethical and socio cultural issues. In: Vayena E; Rowe $\mathrm{PJ}$; Griffin PD; (eds) Current practices and controversies in Assisted Reproduction. Generva, WHO. 2002: 3140.

51. Akande EO. Affordable Assisted Reproductive Technologies in developing cuntries: PRO and CONS: Hum. Reprod. ESHE Monograph 2008: 12-14.

52. Nachtigali RD: International disparities in access to Infertility services. Fertil. Steril. 2006: 85; 871-875.

53 Shah I. Treatment of infertility: an integral part of reproductive health and a necessity: Reprod. Health matter. 1994: 4: 96-97. 
54. United Nations International Conference on population and development Cairo Egypt, 1994.

55 Ombelet W. False perception and common misunderstandings surrounding the subject of infertility in developing countries. Hum. Reprod. ESHE monograph 2008: 811.

56. Edwards L, Olatunbosun $\mathrm{OA}$. the case against New Reproductive technologies (letters to the editors) Br. J. Obstet. Gynaecol. 1997: 104; 969

57. Daar AS, Merali Z. Infertility and social suffering: The case of ART in Developing countries. In: Vayena $\mathrm{E}$, Rowe PJ, Griffin PD (eds) Current practice and controversies in ART. Geneva W.H.O; 2002: 1121.

58. Popovic - Todorovic B, Loft A, Gindhard A, Bangsboll, S; Anderson AM, Anderson AN. A prospective study of predictive factor of ovarian response in standard IVF/ICSI patients treated with recombinant (E.S.H.A.) suggestion for a recombinanat $\mathrm{FSH}$ dosage Normogram. Hum. Reprod. 2003: 118: 781-787.

59. European and Istreali group on highly purified menotropin versus recombinant folliclestimulating hormone. Efficaccy and safety of highly purified menotropin versus recombinant F.S.H in Invitro Fertilisation intracytoplasmic sperm injection cycles randomised comparative trial. Fertil. Steril. 2002. 78: 520-528.

60. Van de Pas MMC, Weima S, Looma, CWN, Broekmans. FJM. The use of fixed distance Embryo transfer after IVF/ICSI equalizes the success rate among physicians. Hum Reprod. 2003: 18: 774-780.

61. Wood EG, Batzer FR, Go KJ, Gutmann JN, Corson SL. Ultransound guided soft catheter Embryo transfer will improve pregnancy rate in in vitro fertilisation. Hum. Reprod. 2005: 15: 107-112.

62. Orhue $A A E$, Aziken $M E$, Ibadin K. IVF/ICSI in a public hospital setting in Nigeria: Practice problems and prospects. 2008 In press.

63. Bentick $B$, Shaw RM, Iffland. CA, Burford G, Bernard A. A randomised comparative study of purified F.S.H and human menopausal gonadotrophin after pituitary desensitation with Buserelin for super ovulation and in vitro fertilisation. Fertil. Steril 1988: 50: 79-84.

64. Olatunbosun OA. et al: Early experience with in vitro fertilisation-embryo transfer and gamette intra fallopian transfer in a Nigeria Hospital. Int. J. Gynaecol. Obstet. 1990: 33: 159-163.

65 Kilani Z, Daklkak A, Ghunaim, S, Gognignis S, Tebarellic Parmegiani L, Fulicori M. A prospective randomised controlled trial comparing highly 
purified hMG with recombinant F.S.H in women undergoing ICSI: Ovarian response and clinical outcomes. Hum. Reprod. 2003: 18: 1194-1199.

66. Lainas L, Petsas G, Stavroponlou G, Alexopoulou E, lladis G, Miraretzis D. Administration of methyl predmisolone to prevent severe ovarian Hyperstimulation syndrome in patients undergoing in vitro fertilisation. Fertil. Steril. 2002: 78: 529-533.

67. Giwa-Osagie OF. The need for infertility services in the developing world: the W.H.O. point of view. Gynaecol. Obstet. Invest. 2004: 57: 58.

68. Ombelet W, Campo R. Affordable I.V.F. for developing coutries. Reprod. Biomed. Online 2007: 15: 257-265. 